\title{
On the generalized bilinear differential equations
}

\author{
${ }^{1}$ M. Y. Adamu, ${ }^{2}$ E. Suleiman \\ ${ }^{1,2}$ Mathematical Sciences Programme, Abubakar Tafawa Balewa University, Bauchi, Nigeria.
}

\begin{abstract}
By using the generalized Hirrota bilinear operators a kind of bilinear differential equations is established and examined when the linear super position principle can apply to the resulting generalized bilinear differential equations. Examples of generalized bilinear differential equations together with an algorithm using weights are computed using a $1+1$ and $2+1$ dimensional equations in order to shed more lights on the presented general scheme for the construction of the bilinear differential equations which posses linear subspaces of solutions.
\end{abstract}

\section{Introduction}

Integrable systems and nonlinear evolution equations have attracted much attention of mathematicians as well as physicist for the last 2 decades. The analysis of exact travelling wave solutions to nonlinear evolution equations plays a vital role in the study of nonlinear physical phenomena. Single solitons are most beneficial solutions among travelling wave solutions. The existence of multi-soliton solutions, especially two-soliton and three-solton is useful in establishing optical communication systems. However, besides solitons, another attractive set of multi-exponential wave solutions [1] is a linear combination of exponential waves. It was shown that some of the nonlinear equations can posses such a linear superposition principle[2,3]. Also, special solutions by combining exponential functions and trigonometric functions were presented and called complexitons [4].

A variety of powerful methods have been used to study integrable systems and nonlinear evolution equations, such as Hirota bilinear method [5], the pfaffian technique, the dressing method [6], the inverse scattering method,the Backlund transformation method [8,9], the Darboux transformation and the generalized symmetry method [10]. These approaches possess powerful features that make it possible to create multiple soliton solutions for a wide range of integrable systems and nonlinear evolution equations.

Many important equations of mathematical physics are rewritten in the Hirota bilinear form through dependent variable transformations [5]. For example the KdV equation:

The Jimbo-Miwa equation

$$
u_{x x x}+6 u u_{x}+u_{t}=0
$$

which can express as

$$
u_{x x x y}+3 u_{y} u_{x x}+3 u_{x} u_{x y}+2 u_{y t}-3 u_{x z}=0
$$

$$
\left(D_{x}^{4}+D_{t} D_{x}\right) F \cdot F=0
$$

under the transformation $u=2(\ln f)_{x x}$

$$
\left(D_{x}^{3} D_{y}+2 D_{t} D_{y}-3 D_{x} D_{z}\right) f \cdot f=0
$$

under the transformation $u=2(\ln f)_{x x}$ respectively, where $D_{x}, D_{y}$, and $D_{t}$ are Hirota bilinear operators and are generally defined by:

$$
D_{x}^{n} D_{t}^{m} D_{y}^{k} f . g=\left(\frac{\partial}{\partial x}-\frac{\partial}{\partial x^{\prime}}\right)^{n}\left(\frac{\partial}{\partial y}-\frac{\partial}{\partial y^{\prime}}\right)^{m}\left(\frac{\partial}{\partial t}-\frac{\partial}{\partial t^{\prime}}\right)^{k} g(x, y, t) f\left(x^{\prime} y^{\prime}, t^{\prime}\right) \mid x=x^{\prime}, t=t^{\prime} y=y^{\prime}
$$

for nonnegative integers $\mathrm{m}, \mathrm{n}$ and $\mathrm{k}$

In this paper we would like tofurther confirm the kind of generalized bilinear differential operator and examine when is the linear superposition principle will be applied to the corresponding bilinear differential equations that is established in [11]. The resulting theory paves a way to construct a new kind of bilinear differential equations which possess linear subspaces of solutions. The considered solutions are linear combinations of exponential travelling wave solutions, and the involved exponential wave solutions may or may not satisfy the corresponding dispersion relations. All the obtained results will exhibit that there are bilinear differential equations different from Hirota bilinear equations, which share some common features with the linear differential equations. 
The paper is organized as follows. In section 2, we will visit the generalized Hirota bilinear operators as introduced [11], and see how we can establish a kind of generalized bilinear differential equations. The analyzed linear superposition principle for exponential travelling waves and the established criterion for guaranteeing the existence of linear subspaces of exponential travelling wave solutions to the generalized bilinear differential equations is employed as in [11].while in section four we will present two examples of newly introduced bilinear differential equations, together with an algorithm using weights to compute. Finally, concluding remarks will be drawn in section 5 .

\section{Bilinear Differential Operators And Equations}

Let $\mathrm{M}, \mathrm{P} \in \mathrm{N}$ be given,. We introduce a kind of bilinear differential equation:

$$
D_{p_{j} x_{1}}^{n_{1}} \ldots D_{p_{j} x_{M}}^{n_{M}} f . g=\left.\prod_{i=1}^{M}\left(\frac{\partial}{\partial x_{i}}+\alpha \frac{\partial}{\partial x_{i}^{\prime}}\right)^{n_{i}} f\left(x_{1} \ldots x_{m}\right) g\left(x_{1}^{\prime} \ldots x_{M}^{\prime}\right)\right|_{x_{1}^{\prime}=x, x_{M}^{\prime}=x_{M}}
$$

Where $n_{1} \ldots n_{M}$ are arbitrary nonnegative integers and for an integer $\mathrm{m}$ the mth power of $\alpha$ is defined by:

$\alpha^{m}=(-)^{r(m)}$, if $m \equiv r(m) \bmod p$, with $0 \leq r(m)<p$

For example if $\mathrm{p}=2 \mathrm{k}(\mathrm{k} \in \mathrm{N})$, All the above bilinear differential operators are Hirota bilinear operators, since $D_{2 k, x}=D_{x}$ (see for example [11]) for the detailed analysis of the algorithm)

Ie if $\mathrm{p}=2$, then we have

$\alpha=-1, \alpha^{2}=1, \alpha^{3}=-1, \alpha^{4}=1, \ldots$

which gives the pattern of symbols for the Hirota bilinear operators

$-,+,-,+,-,+,-,+, \ldots(p=2)$

And if $\mathrm{p}=3$, we have

$\alpha=-1, \alpha^{2}=1, \alpha^{3}=1, \alpha^{4}=-1, \alpha^{5}=1, \alpha^{6}=1, \ldots$

which gives the pattern of symbols for the Hirota bilinear operators

$-,+,+-,+,+,-,+,+, \ldots(p=3)$

And if $\mathrm{p}=5$, we have

$\alpha=-1, \alpha^{2}=1, \alpha^{3}=-1, \alpha^{4}=1, \alpha^{5}=1, \alpha^{6}=-1, \alpha^{7}=1, \alpha^{8}=-1, \alpha^{9}=1, \alpha^{10}=1, \ldots$

which gives the pattern of symbols for the Hirota bilinear operators

$-,+,-,++,-,+,-,+,+, \ldots(\mathrm{p}=5)$

And when $\mathrm{p}=7$, the pattern of symbol is

$-,+,-,+,-++,-,+,-,+,-,+,+, \ldots(p=7)$

Following those patterns of symbols, some new bilinear differential operators can be computed as:

$D_{3, x} f \cdot g=f_{x} g-f g_{x}$

$D_{3, x} D_{3, t}=f_{x t} g-f_{x} g_{t}-f_{t} g_{x}+f g_{x t}$

$D_{3, x}^{3} f . g=f_{x x x} g-3 f_{x x} g_{x}+3 f_{x} g_{x x}+f g_{x x x}$

$D_{3, x}^{2} D_{3, t} f g=f_{x x t} g-f_{x x} g_{t}-2 f_{x t} g_{x}++2 f_{x} g_{x t}+f_{t} g_{x x}+f g_{x x t}$

$$
D_{3, x}^{3} D_{3, t} f g=f_{x x x t} g-f_{x x x} g_{t}-3 f_{x x t} g_{x}+3 f_{x t} g_{x x}+3 f_{x x} g_{x t}+3 f_{x} g_{x x t}+f_{t} g_{x x x}-f g_{x x x t}
$$

$D_{3, x}^{4} f g=f_{x x x x} g-4 f_{x x x} g_{x}+6 f_{x x} g_{x x}+4 f_{x} g_{x x x}-f g_{x x x x}$

$D_{3, x}^{5} f \cdot g=f_{x x x x x} g-5 f_{x x x x} g_{x}+10 f_{x x x} g_{x x}+10 f_{x x} g_{x x x}-5 f_{x} g_{x x x x}+f g_{x x x x x}$

$D_{3, x}^{6} f . g=f_{x x x x x x} g-6 f_{x x x x x x} g_{x}+15 f_{x x x x} g_{x x}+20 f_{x x x} g_{x x x}-15 f_{x x} g_{x x x x}+6 f_{x} g_{x x x x x x}+f g_{x x x x x x}$ 


$$
\begin{aligned}
& D_{5, x} f \cdot g=f_{x} g-f g_{x} \\
& D_{5, x} D_{5, t}=f_{x t} g-f_{x} g_{t}-f_{t} g_{x}+f g_{x t} \\
& D_{5, x}^{3} f . g=f_{x x x} g-3 f_{x x} g_{x}+3 f_{x} g_{x x}-f g_{x x x} \\
& D_{5, x}^{2} D_{5, t} f . g=f_{x x t} g-f_{x x} g_{t}-2 f_{x t} g_{x}+2 f_{x} g_{x t}+f_{t} g_{x x}-f g_{x x t} \\
& D_{5, x}^{3} D_{3, t} f . g=f_{x x x t} g-f_{x x x} g_{t}-3 f_{x x t} g_{x}+3 f_{x t} g_{x x}+3 f_{x x} g_{x t}-3 f_{x} g_{x x t}-f_{t} g_{x x x}+f g_{x x x t} \\
& D_{5, x}^{5} f . g=f_{x x x x} g-5 f_{x x x x} g_{x}+10 f_{x x} g_{x x x}+5 f_{x} g_{x x x x}+f g_{x x x x}
\end{aligned}
$$

We can observe that from those formulas we see that except for $D_{5, x}^{3} f . f=0, D_{3, x}^{3} f . f$, $D_{3, x}^{5} f . f$ and $D_{5, x}^{5} f . f$ do not equal zero, which is different from the Hirota case: $D_{x}^{3} f . f=D_{x}^{5} f . f=0$ Now let $\mathrm{p}$ be a polynomial in $\mathrm{M}$ variables and introduce a generalized bilinear differential equation:

$$
P\left(D_{p, x_{1}}, \ldots, D_{p, x_{M}}\right) f . f=0
$$

Observe particularly that when $\mathrm{p}=3$, we have the generalized bilinear KdV equation:

$$
\left(D_{3, x} D_{3, t}+D_{3, x}^{4}\right) f . f=2 f_{x t} f-2 f_{x} f_{t}+6 f_{x x}^{2}=0
$$

The generalized Sawada-Kotera equation:

$$
\left(D_{3, x} D_{3, t}+D_{3, x}^{6}\right) f . f=2 f_{x t} f-2 f_{x} f_{t}+2 f f_{x x x x x}-20 f_{x x x}^{2}=0
$$

We will like, following the approach of $[10,12]$ to discuss the linear subspaces of solutions to the generalized bilinear differential equations defined by (2.3). to be precise, as in Hirota case [5], and also show when the linear superposition principle will apply to the generalized bilinear differential equation (2.3).

\section{Linear Superposition Principle}

Let us now fix $N \in \square$ and in introduce $\mathrm{N}$ waves variables:

$$
\eta_{i}=k_{1, i} x_{1}+\ldots+k_{M, i} x_{M}, \quad 1 \leq i \leq N
$$

and $N$ exponential wave functions:

$$
f_{i}=e^{\eta_{i}}=e^{k_{1, i} x_{1}+\ldots+k_{M, i} x_{M}}, \quad 1 \leq i \leq N
$$

Where the $k_{j, i}{ }^{\prime} s$ are constants. Note also that we have a bilinear identity:

$$
P\left(D_{p . x_{1}}, \ldots, D_{p, x_{M}}\right) e^{\eta_{i}} \cdot e^{\eta_{j}}=P\left(k_{1, i}+\alpha k_{1, j}, \ldots, k_{M, i}+\alpha k_{M, j}\right) e^{\eta_{i}}+e^{\eta_{j}},
$$

Where the power of $\alpha$ obey the rule (2.2). Then we consider a linear combination solution to the linear differential equation (2.3):

$$
f=\varepsilon_{1} f_{1}+\ldots+\varepsilon_{N} f_{N}=\varepsilon_{1} e^{\eta_{1}}+\ldots+\varepsilon_{N} e^{\eta_{N}},
$$

Where $\varepsilon_{i}, 1 \leq i \leq N$, are arbitrary constants. Using (3.3) we can compute that 


$$
\begin{aligned}
& P\left(D_{p, x_{1}}, \ldots, D_{p, x_{M}}\right) f . f \\
= & \sum_{i, j=1}^{N} \varepsilon_{i} \varepsilon_{j} P\left(D_{p, x_{1}}, \ldots, D_{p, x_{M}}\right) e^{\eta_{i}} \cdot e^{\eta_{j}} \\
= & \sum_{i, j=1}^{N} \varepsilon_{i} \varepsilon_{j} P\left(k_{1, i}+\alpha k_{1, j}, \ldots, k_{M . i}+\alpha k_{M, j}\right) e^{\eta_{i}+\eta_{j}} \\
= & \sum_{i=1}^{N} \varepsilon_{i}^{2}\left[P\left(k_{1, i}+\alpha k_{1, j}, \ldots, k_{M . i}+\alpha k_{M, j}\right) e^{2 \eta_{i}}\right. \\
& +\sum_{1 \leq i<j \leq N} \varepsilon_{i} \varepsilon_{j}\left[P\left(k_{1, i}+\alpha k_{1, j}, \ldots, k_{M . i}+\alpha k_{M, j}\right)\right. \\
& +P\left(k_{1, i}+\alpha k_{1, j}, \ldots, k_{M . i}+\alpha k_{M, j}\right) e^{\eta_{i}+\eta_{j}}
\end{aligned}
$$

It thus follows that a linear combination function $\mathrm{f}$ defined by (3.4)solves the generalized bilinear differential equations (2.3) if and only if:

$$
P\left(k_{1, i}+\alpha k_{1, j}, \ldots, k_{M, i}+\alpha k_{M, j}\right)+P\left(k_{1, j}+\alpha k_{1, i}, \ldots, k_{M, j}+\alpha k_{M, i}\right)=0, \quad 1 \leq i \leq j \leq N,
$$

are satisfied, where the powers of $\alpha$ obey the rule (2.2). the condition in (3.6) present a system of nonlinear algebraic equations on the wave related numbers $k_{j, i}{ }^{\prime} s$ and the coefficients of the polynomial P. generally, it is not easy to solve (3.6). but in many cases, such systems have various sets of solutions .

However, the whole result was summarized in a theorem (criterion for linear superposition principle) in [13]. The theorem tells us that the linear superposition principle can apply to generalized bilinear differential equations defined by (2.3). it also paves a way of constructing $\mathrm{N}$-wave solutions to the generalized bilinear differential equations.

\section{Application}

Let us now compute examples of the generalized bilinear differential equations, defined by (2.3), with linear subspaces of solutions, by applying the theorem in [11] . The problem is how to construct a multivariate polynomial $P\left(x_{1}, \ldots, x_{M}\right)$ such that:

$$
P\left(k_{1,1}+\alpha k_{1,2}, \ldots, k_{M, 1}+\alpha k_{M, 2}\right)+P\left(k_{1,2}+\alpha k_{1,1}, \ldots, k_{M, 2}+\alpha k_{M, 1}\right)=0,
$$

Holds for two sets of constants $k_{1, i}, \ldots, k_{M, i}, i=1,2$, where the powers of $\alpha$ obey the rule (2.2). our basic idea is to introduce weights of independent variables and then use parameterization of wave numbers and frequencies.

Following the approach of [11], we introduce the weights for the independent variables:

$$
\left(w\left(x_{1}\right), \ldots, w\left(x_{M}\right)\right)=\left(n_{1}, \ldots, n_{M}\right)
$$

Where each weight is an integer, and then form a polynomial $P\left(x_{1}, \ldots, x_{M}\right)$ being homogenous in some weight. Second, for $\mathrm{i}=1,2$, we parameterization the constants $k_{1, i}, \ldots, k_{M, i}$, consisting of wave numbers and frequencies, using a free parameter $k_{i}$ as follows:

$$
k_{j, i}=b_{j} k_{i}^{n_{i}}, 1 \leq j \leq M
$$

Where the $b_{j}{ }^{\prime} s$ are constants to be determined. The parameterization balances the degree of the free parameters in the system (4.1). detailed procedures could also be found in [11]

In what follows we present two illustrative examples in a $1+1$ and a $2+1$ dimensions, which apply to the above parameterization achieved by using one free parameter.

\section{Example1. Examples with positive weights:}

Let us introduce the weights of independent variables:

$(\mathrm{w}(\mathrm{x}), \mathrm{w}(\mathrm{t}))=(1,2)$

Then a general even polynomial being homogenous in weights 4 reads:

$$
P=c_{1} x^{4}+c_{2} x^{2}+c_{3} t^{2}
$$

Following the parameterization of wave numbers and frequency in (4.3), the wave variables read 
$\eta_{i}=k_{i} x+b_{1} k_{i}^{3} t, 1 \leq i \leq N$,

Where the $k_{i}, 1 \leq i \leq N$ are arbitrary constants, but $b_{1}$ is a constant to be determined. In this example, the corresponding generalized bilinear differential equation reads:

$P\left(D_{3, x}, D_{3, t}\right) f . f=6 c_{1} f_{x x}^{2}+2 c_{2} f_{x x t} f+2 c_{3} f_{t t} f-2 c_{3} f_{t}^{2}=0$

(4.6)

And the corresponding linear subspace of $\mathrm{N}$-wave solutions is given by:

$f=\sum_{i=1}^{N} \varepsilon_{i} f_{i}=\sum_{i=1}^{N} \varepsilon_{i} e^{k_{i} x+b_{1} k_{i}^{2} t}$,

Where $\varepsilon_{i}, 1 \leq i \leq N$ are arbitrary constants, but $b_{1}$ need to satisfy:

$$
\left.\begin{array}{l}
3 c_{1}-c_{3} b_{1}^{2}=0 \\
c_{2} b_{1}+c_{3} b_{1}^{2}=0
\end{array}\right\}
$$

Therefore, the coefficients of the polynomial P need to satisfy:

$c_{2}^{2}=3 c_{1} c_{3}$

Then the non-trivial solution of $b_{1}$ is given:

$b_{1}=\frac{-3 c_{1}}{c_{2}}$

\section{Example 2}

Let us introduce the weight of independent variables:

$$
(\mathrm{w}(\mathrm{x}), \mathrm{w}(\mathrm{y}), \mathrm{w}(\mathrm{t}))=(1,3,2)
$$

Then an even polynomial being polynomial in weight 3 reads:

$$
P=c_{1} x^{3}+c_{2} y+c_{3} x t
$$

Following the parameterization of wave numbers and frequency in (4.3), the wave variables $\operatorname{read} \eta_{i}=k_{i} x+b_{1} k_{i}^{3} y+b_{2} k_{i}^{2} t, \quad 1 \leq i \leq N$,

Where the $k_{i}, 1 \leq i \leq N$ are arbitrary constants, but $b_{1}$ and $b_{2}$ are constants to be determined. In this example, the corresponding generalized bilinear differential equation reads:

$$
P\left(D_{3, x}, D_{3, y}, D_{3, t}\right) f . f=2 c_{1} f_{x x x} f+2 c_{3} f_{x t} f-2 c_{3} f_{x} f_{t}=0
$$

the corresponding linear subspace of $\mathrm{N}$-wave solutions is given by:

$$
f=\sum_{i=1}^{N} \varepsilon_{i} f_{i}=\sum_{i=1}^{N} \varepsilon_{i} e^{k_{i} x+b_{1} k_{i}^{3} y+b_{2} k_{i}^{2} t}
$$

Where $\varepsilon_{i}, 1 \leq i \leq N$ are arbitrary constants, but $b_{1}$ need to satisfy:

$$
\left.\begin{array}{l}
c_{1}-c_{3} b_{2}+c_{2} b_{1}=0 \\
c_{3} b_{2}-c_{2} b_{1}=0
\end{array}\right\}
$$

if $c_{1}=0$, then the solution becomes:

$$
b_{1}=c_{3}, b_{2}=c_{2}
$$

(4.16)

\section{Examples with positive and negative weights}

\section{Example 1}

Let us introduce the weight of independent variables:

$$
(\mathrm{w}(\mathrm{x}), \mathrm{w}(\mathrm{t}))=(1,-2)
$$

Then an even polynomial being polynomial in weight 3 reads:

$$
P=c_{1} x+c_{2} x^{3} t
$$


Following the parameterization of wave numbers and frequency in (4.3), the wave variables $\operatorname{read} \eta_{i}=k_{i} x+b_{1} k_{i}^{-2} t, \quad 1 \leq i \leq N$,

Where the $k_{i}, 1 \leq i \leq N$ are arbitrary constants, but $b_{1}$ is a constant to be determined. In this example, the corresponding generalized bilinear differential equation reads:

$P\left(D_{3, x}, D_{3, t}\right) f \cdot f=6 c_{2} f_{x x} f_{x t}=0$

The corresponding linear subspace of $\mathrm{N}$-wave solutions is given by:

$f=\sum_{i=1}^{N} \varepsilon_{i} f_{i}=\sum_{i=1}^{N} \varepsilon_{i} e^{k_{i} x+b_{2} k_{i}^{-2} t}$

Where $\varepsilon_{i}, 1 \leq i \leq N$ are arbitrary constants, but $b_{1}$ need to satisfy

$6 c_{2} b_{1}=0$

Which gives $c_{2}=0$

While $b_{1}$ is arbitrary and can be written as:

$$
b_{1}=c_{1}
$$

Example 2:

Introducing the weights of independent variables as:

$(\mathrm{w}(\mathrm{x}), \mathrm{w}(\mathrm{y}), \mathrm{w}(\mathrm{t}))=(1,-1,2)$

Then a general even polynomial being homogenous in weights 3 reads:

$P=c_{1} x^{3}+c_{2} x^{4} y+c_{3} x t+c_{4} y t^{2}$

Following the parameterization of wave numbers and frequency in (4.3), the wave variables read $\eta_{i}=k_{i} x+b_{1} k_{i}^{-1} y+b_{2} k_{i}^{2} t, \quad 1 \leq i \leq N$,

Where the $k_{i}, 1 \leq i \leq N$ are arbitrary constants, but $b_{1}$ and $b_{2}$ are constants to be determined. In this example, the corresponding generalized bilinear differential equation reads:

$$
\begin{aligned}
P\left(D_{3, x}, D_{3, y}, D_{3, t}\right) f . f= & 2 c_{1} f_{x x x} f+2 c_{2} f_{x x x x y} f-8 c_{2} f_{x x x y} f_{x}+12 c_{2} f_{x x y} f_{x x}+8 c_{2} f_{x x x} f_{x y} \\
& -2 c_{2} f_{x x x} f_{y}+2 c_{3} f_{x t} f-2 c_{3} f_{x} f_{t}+2 c_{4} f_{t t t y} f=0
\end{aligned}
$$

And the corresponding linear subspace of $\mathrm{N}$-wave solutions is given by:

$f=\sum_{i=1}^{N} \varepsilon_{i} f_{i}=\sum_{i=1}^{N} \varepsilon_{i} e^{k_{i} x+b_{1} k_{i}^{-1} y+b_{2} k_{i}^{2} t}$,

Where $\varepsilon_{i}, 1 \leq i \leq N$ are arbitrary constants, but $b_{1}$ and $b_{2}$ need to satisfy

$$
\left.\begin{array}{r}
c_{4} b_{1} b_{2}^{2}+c_{3} b_{2}+6 c_{2} b_{1}=0 \\
c_{1}+3 c_{2} b_{1}=0 \\
-c_{3} b_{2}-3 c_{2} b_{1}=0
\end{array}\right\}
$$

which gives a solution of

$$
b_{1}=-\frac{c_{1}}{3 c_{2}}, b_{2}=\frac{c_{1}}{c_{3}}
$$

$c_{1}^{2} c_{4}=-3 c_{2} c_{3}^{2}$

\section{Conclusion}

We were able to ascertain newly introduced kind of bilinear differential operators established in [10] and analyzed when the corresponding generalized bilinear equation posses superposition principle. In particular we computed two examples using $1+1$ and a $2+1$ dimensional equation by an algorithm using weights and their 
linear subspaces of exponential travelling wave solutions. The balance requirements of the weights allow us to present a class of parameterization of wave numbers and frequencies.

Our results further confirm the generalized Hirota bilinear operators and the established bridge between bilinear differential equations and linear differential equations [11]. The existence of linear subspaces of solutions amends the diversity of exact solution generated by various analytical methods (see for example, [5]. The generalized bilinear operators (2.1), definitely bring more chance to generate non trivial trilinear differential equations.

\section{References}

[1] Ma, W. X., Huang,T. W. Zhang, Y., A multiple Exp-function Method for nonlinear differential equations and its application Physica Scipta 822010065003.

[2] Ma, W. X. and Fan, E., Linear superposition principle applying to Hirota bilinear equations. Computers And Mathematics With Appllications, 61,(2011 950-959

[3] W. X., Ma, Y .Zhang,, Y .Tang,.. and J . Tu,. Hirota bilinear equations with linear subspaces of solutions. Appllied Mathematics And Computations 218, 2012, 7174-7183

[4] W. X, Ma, , and Y. You,Solving Korteweg de-Vries equation by its bilinear form: Wronskian solution. Trans. Amer. Math. Soc., 357,2005:1753-1778

[5] R. Hirota.. The Direct Method in Soliton Theory. (Cambridge University Press) (2004)

[6] Asaad, M. G and Ma, W. X., , Pfaffian solution to a (3+1)-dimensional generalized B.typeKadomtsev-Petviashvilli equation and its modified counterpart, applied Math. And Comp.218, 2012, 5524-5542

[7] R. Hirota,., a new form of Baclund Transformation and its relation to the inverse scattering problem. Progr. Of Theoret. Phys., 52 , 1974, 329-338

[8] N. C, Freeman, and J.J.C Nimmo, the use of Backlund transformation in obtaining N-soliton solutions in Wronskian form. Phys. lett. A $95,1983,1-3$

[9] S . Zangh, and T. C, Xia,., A generalized new auxiliary equation and its application to nonlinear partial differential equations. Phys. Lett. A, 363, 2007, 356-360

[10] M. Jimbo. and T. Miwa, solitons and infinite dimensional Lie algebra, publications in research Institute for Mathematical Sciences. 19, 1983, 943-1001

[11] W. X. Ma Generalized bilinear differential equations Diversity of exact solution to a restricted Boiti-Leon-pempinelli dispersive long wave system. Physics Letter. A 310 2012,325-333

[12] A. Wazwaz, multiple soliton solutions for Calogero-Bogoyavlensskii-Schiff, Jimbo-Miwa and YTSF equations, Appllied Mathematics and Computations 203, 2008, 592-597.

[13] M. Y. Adamu, and E. Suleiman,. linear subspaces of solutions applied to Hirota bilinear equations, Aceh, Journ. Sci. and Techn.1 (2) $2012,45-51$ 